\title{
Maximum Magnitude of Seismicity Induced by a Hydraulic Fracturing Stage in a Shale Reservoir: Insights from Numerical Simulations
}

\author{
Amanda M. M. Bustin, R. Marc Bustin \\ Department of Earth, Ocean and Atmospheric Sciences, The University of British Columbia, Vancouver, Canada \\ Email: abustin@eoas.ubc.ca
}

How to cite this paper: Bustin, A.M.M. and Bustin, R.M. (2020) Maximum Magnitude of Seismicity Induced by a Hydraulic Fracturing Stage in a Shale Reservoir: Insights from Numerical Simulations. Engineering, 12, 516-533.

https://doi.org/10.4236/eng.2020.127036

Received: January 15, 2020

Accepted: July 25, 2020

Published: July 28, 2020

Copyright ( 2020 by author(s) and Scientific Research Publishing Inc. This work is licensed under the Creative Commons Attribution International License (CC BY 4.0).

http://creativecommons.org/licenses/by/4.0/

\begin{abstract}
A key unknown limiting assessment of risk posed by inducing anomalous seismicity during hydraulic fracturing is the potential maximum magnitude of an event. To provide insights into the variation in maximum magnitude that can be induced by a hydraulic fracturing stage, worst-case scenarios were simulated in $2 \mathrm{D}$ using coupled hydro-geomechanical models. The sensitivity of the magnitude to the hydro-geomechanical properties of the fault and matrix rock were quantitatively compared through parametric analysis. Our base model predicts a maximum event with moment magnitude $\left(M_{w}\right) 4.31$ and $M_{w}$ values range from 3.97 to 4.56 for the series of simulations. The highest magnitude is predicted for the model with a longer fault and the lowest magnitude for the model with a smaller Young's modulus. For our models, the magnitude is most sensitive to changes in the Young's modulus and length of the fault and least sensitive to changes in the initial reservoir pressure (i.e. pore pressure) and the Poisson's ratio.
\end{abstract}

\section{Keywords}

Induced Seismicity, Maximum Magnitude, Hydraulic Fracturing, Gas Shales, Hydro-Geomechanical Modelling

\section{Introduction}

The potential risk to the environment and public health and safety resulting from anomalous seismicity induced by hydraulic fracturing has become an increasing concern since the first reported occurrence in 2011 from Blackpool, England [1] [2] [3]. While the research effort has increased with the concern, various challenges still exist to accurately assess the true risk posed by anomal- 
ous seismicity induced by hydraulic fracturing. One of the key challenges, as well as a subject of considerable debate, is the maximum magnitude of an event that can be induced by a particular hydraulic fracturing operation. Some researchers have suggested that an upper limit exists for the maximum magnitude, which is controlled by the fluid injection operation [4] [5] [6] [7]. Other researchers argue that a deterministic upper bound is only applicable for self-arresting ruptures, whereas in high-stress environments, the rupture may be self-sustaining and the maximum magnitude is only bound by tectonics [8] [9].

In an attempt to better understand maximum magnitude, a parametric analysis was performed to quantify the sensitivity of the magnitude to variations in the hydro-geomechanical properties of the fault and matrix rock. In this study, the fluid injection from a hydraulic fracturing stage is simulated for a series of numerical models with a critically stressed fault within a fractured shale reservoir using the 2D discontinuum based distinct element model, UDEC [10]. The input parameters for the modelling were chosen to represent a worst-case scenario, providing insight into the maximum magnitude earthquake that can be induced by a hydraulic fracturing stage. The fluid injection rate and duration, length of the fault, distance from fault to injection source, maximum and minimum principal stresses, pore pressure, and the Poisson's ratio and Young's modulus of the shale, were systematically varied to end-member values for the parametric analysis. Understanding the sensitivity of the magnitude of induced events will aid in developing protocols for reducing the probability of inducing felt events and mitigation procedures to prevent larger magnitude induced events, as well as help to understand the variations in the magnitude of induced seismicity between regions. Following a brief explanation of the modelling procedure, the results of the simulations are presented, compared, and discussed.

\section{Model}

In order to simulate the interaction between natural and hydraulic fractures in combination with fault reactivation during a hydraulic fracturing stage, a coupled hydro-geomechanical approach is necessary. The two-dimensional (2D) distinct element model, UDEC version 5.0 (UDEC5, first presented by [11]), which uses the discontinuum, distinct-element method, was chosen for this study as it is specially designed to simulate fluid flow and the resulting behavior of fractured rock masses. The distinct-element method uses an explicit time-stepping algorithm, which allows for the deformation of the rock matrix blocks and large shear and closure/dilation displacements along the discontinuities between blocks.

In UDEC, Newton's equations of motion are directly solved using explicit time-stepping. The $2 \mathrm{D}$ model space is discretized into mutually interacting blocks (i.e. discrete elements) to simulate a discontinuous system with deformable boundaries representing planes for possible failure (i.e. fractures/joints/bedding planes/faults). Both shear and normal displacements are allowed along the 
boundaries according to the Mohr-Coulomb slip model with residual strength. The blocks are deformable and subdivided into a mesh of finite difference elements controlled by the basic Mohr-Coulomb slip criterion. UDEC5 performs a coupled hydro-geomechanical analysis with fluid flow through the discontinuities, while the blocks are impermeable. The program enables quantification of shear slip and dilation along pre-specified failure planes caused by disturbance of the initial stress field resulting from fluid injection and the generation of hydraulic fractures. Therefore, the response of the modeled system to fluid injection includes the mechanical effects, due to the changing stress field, coupled with the flow effects, due to changes in fluid pressure.

In this study, the pore pressure build-up and diffusion along a fault, near the injection source, within jointed shale, were simulated and the resulting slip along the fault was interpreted as a seismic event. The moment magnitude $\left(M_{w}\right)$, calculated from the magnitude and extent of the slip, was then quantitatively compared for a series of simulations. The input parameters that were investigated during the parametric analysis include: the injection duration and flow rate; the length of the fault and its distance from the injection source; the magnitudes of the maximum and minimum principal stresses and the pore pressure; and the Young's modulus and Poisson's ratio of the shale matrix. Each parameter was systematically varied from the average value in the base model to the maximum and minimum end-member values shown in Table 1.

The input parameter values for our simulation were chosen to represent a possible worst-case scenario where the injected fluid is transported through the newly created hydraulic fractures and opened fracture network to a nearby, critically stressed fault. A 2D numerical model was built, $3 \times 3 \mathrm{~km}$ in size, containing an orthogonal incipient fracture network, oriented to the principal stress directions, with a $50 \mathrm{~m}$ spacing and a fault oriented $30^{\circ}$ from the maximum principal stress. The 2D model, which is shown in Figure 1, represents a horizontal plane with a strike-slip fault. The initial friction angle of the fractures was set as $30^{\circ}$, the cohesion $1 \mathrm{MPa}$, and the tensile strength $0.5 \mathrm{MPa}$. Strain-softening was included in the models by setting the residual friction angle to $25^{\circ}$, while the residual cohesion and tensile strength are 0 . The fault was modeled having no cohesion, tensile strength, or residual friction and an initial friction angle of $20^{\circ}$. The large difference between the residual and peak friction angles results in the brittle behavior of the fault. To allow sufficient fluid flow to model a worst-case scenario, the peak permeability of the fractures was input as $1 \mathrm{md}$, with a minimum value of $0.1 \mathrm{md}$ and the peak permeability of the fault was input as $1 \mathrm{~d}$, with a minimum value of $100 \mathrm{md}$. The density of the matrix blocks in the model was held constant at $2.5 \mathrm{~g} / \mathrm{cc}$ throughout the simulations.

For the base model, simulated with the average parameter values, a maximum principal stress of $30 \mathrm{MPa}$, a minimum principal stress of $20 \mathrm{MPa}$, and a pore pressure of $10 \mathrm{MPa}$ were applied. The effective principal stresses (green circle) and the failure envelopes for the incipient fractures and the fault are plotted on a Mohr diagram in Figure 2, showing that the fault is critically oriented for shear failure. 
Table 1. Parameter values for the base model and the values tested during the parametric analysis (in parentheses).

\begin{tabular}{|c|c|c|c|}
\hline Parameter & Fractures & Fault & Block \\
\hline Friction angle $\left({ }^{\circ}\right)$ & 30 & 20 & \\
\hline Residual Friction angle $\left({ }^{\circ}\right)$ & 25 & & \\
\hline Permeability (md) & 1 & & \\
\hline Residual perm (md) & 0.1 & & \\
\hline Cohesion (MPa) & 1 & & \\
\hline Residual cohesion $(\mathrm{MPa})$ & 0 & & \\
\hline Tensile strength (MPa) & 0.5 & & \\
\hline Residual tensile strength (MPa) & 0 & & \\
\hline Normal stiffness (MPa) & $1 \mathrm{E}+04$ & & \\
\hline Shear stiffness (MPa) & $1 \mathrm{E}+03$ & & \\
\hline Spacing $(\mathrm{m})$ & 50 & & \\
\hline Length (m) & & $1000(500,1500,5000)$ & \\
\hline Distance to injector (m) & & $100(50,200,300)$ & \\
\hline Density $(\mathrm{g} / \mathrm{cc})$ & & & 2.5 \\
\hline Young's modulus (GPa) & & & $30(10,50)$ \\
\hline Poisson's ratio & & & $0.25(0.20,0.30)$ \\
\hline Pore pressure $(\mathrm{MPa})$ & & & $10(5,15)$ \\
\hline Max principal stress (MPa) & & & $30(25,35)$ \\
\hline Min principal stress $(\mathrm{MPa})$ & & & $20(15,25)$ \\
\hline Injection rate $\left(\mathrm{m}^{2} / \mathrm{sec}\right)$ & & & $0.1(0.05,0.15)$ \\
\hline Injection time (min) & & & $50(25,75)$ \\
\hline
\end{tabular}

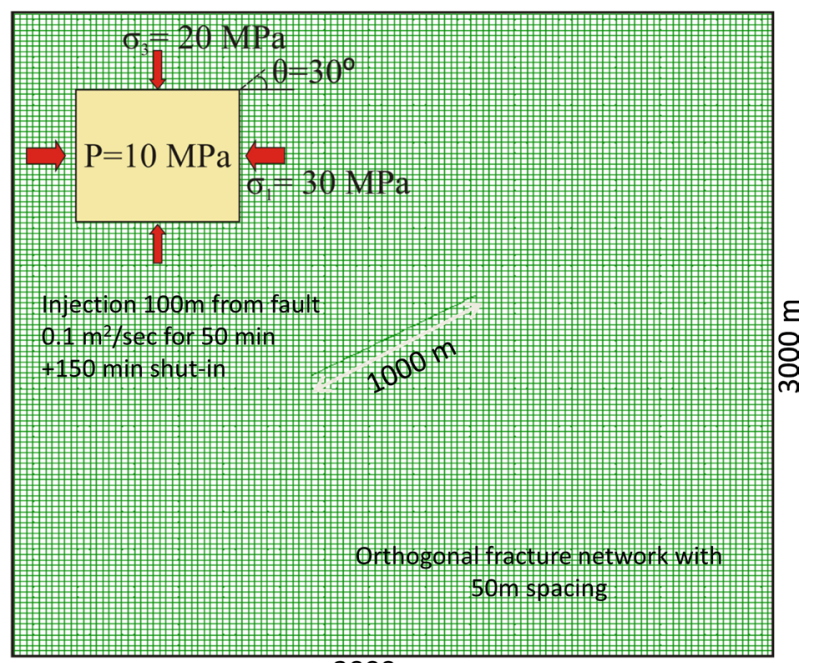

$3000 \mathrm{~m}$

Figure 1. The $3 \times 3 \mathrm{~km}$ model built in UDEC5 with an orthogonal fracture network, aligned with the principal stress directions and spaced at $50 \mathrm{~m}$., and a fault, oriented $30^{\circ}$ from the maximum principal stress. The base model, with average parameters, is shown where the injection source is $100 \mathrm{~m}$ from a $1 \mathrm{~km}$ long fault. 


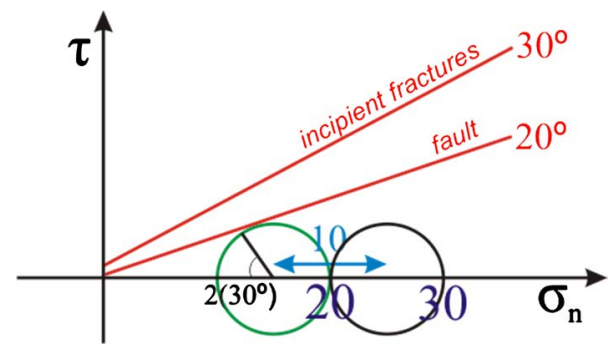

Figure 2. Mohr diagram depicting the stress state of the model and the failure criteria (red curves) for the incipient fractures and the fault. The minimum and maximum principal stresses are plotted on the normal stress axis as the black circle with a diameter equal to the differential stress (i.e. $\sigma_{1}-\sigma_{3}$ ). Subtracting the pore pressure from the principal stresses translates the black circle to the green, effective stress circle. A fault oriented $30^{\circ}$ from the maximum principal stress, which is plotted on the effective stress circle as 2 $\left(30^{\circ}\right)$ (i.e. $60^{\circ}$ ) from the normal stress axis, is on the failure enveloped, indicating it is critically stressed.

The model is first constructed with the orthogonal fracture network and fault, then is compressed to achieve the internal principal stresses, following which fluid is injected. In the base model, fluid is injected from a source $100 \mathrm{~m}$ from the center of a $1 \mathrm{~km}$ long fault at a rate of $0.1 \mathrm{~m}^{2} / \mathrm{s}$ for 50 minutes (note that flow rates are in area per time due to the $2 \mathrm{D}$ model). Following shut in, the models were simulated for a total of 200 minutes, in order to investigate the post-injection response. The average rock mechanical properties of the matrix blocks for the base model were modeled as $30 \mathrm{GPa}$ for the Young's modulus and 0.25 for the Poisson ratio. The parameters input for the base model are summarized in Table 1.

The magnitude earthquake induced by the fluid injection was calculated for each model at 5-minute intervals during the injection period and 10-minute intervals after shut-in by assuming all the shear slip along the fault, since the start of injection, was accommodated instantaneously at that time. At each time interval, the length of the fault that slipped $>1 \mathrm{~cm}$ and the average of the slips along the patches of that fault length (average slip) were determined to calculate a moment magnitude, $M_{w}$ using:

$$
M_{w}=\frac{2}{3} \log M_{0}-6 \quad[12],
$$

where $M_{0}$, the scalar seismic moment in Nm, is the product of the shear modulus of the host rock, $\mu$, the area of the fault that slip, $A$, and the slip along the fault, $s$.

$$
M_{0}=\mu A s \quad \text { [13]. }
$$

The fault area was calculated assuming a circular fault with a diameter equal to the modeled rupture length, $L$ :

$$
A=\pi\left(\frac{L}{2}\right)^{2}
$$

The modeled slip and length of fault slip and the calculated $M_{w}$ were compared for the series of simulations providing insights into the relative importance of the hydro-geomechanical parameters on the maximum possible magni- 
tude earthquake induced by a hydraulic fracturing stage.

While faults within shales are often argued to be impermeable (ex. [14] [15]), the goal of this study is to investigate the range of maximum possible magnitudes possible for varying hydro-geomechanical parameters. Therefore, instead of predicting multiple smaller events along the fault and/or stable slip, as commonly observed during hydraulic fracturing and in numerical modelling, this study assumes a permeable fault in order to predict the worst-case scenario.

The main uncertainty in the modelling is the $2 \mathrm{D}$ simplification, which results in difficulties estimating a representative injection rate and fault plane area. In addition, the fluid flow in UDEC5 is restricted to the discontinuities between blocks and the reservoir is assumed to be fully water saturated with no gas, which also provides limitations to the modelling. Therefore, the goal of this study is not to provide absolute magnitudes, but a comparison of the change in magnitude with hydro-geomechanical parameters.

\section{Results and Discussion}

The simulation of a hydraulic fracturing stage near a critically-stressed fault for the base case model predicts an average of $3.90 \mathrm{~cm}$ of slip (solid curve in Figure 3(a)) along $806 \mathrm{~m}$ of the fault (dotted curved in Figure 3(a)), after 5 minutes of injection, which produces a $M_{w} 3.59$ event (Figure 3(b)). While the average slip increases smoothly with time to a peak at the end of injection, the length of fault rupture increases in a step-function, due to the discretization of the fault in the model. If an event is induced after 50 minutes of injection, the entire $1 \mathrm{~km}$ length of the fault ruptures an average of $30.9 \mathrm{~cm}$, producing a $M_{w} 4.31$ event. The length of fault rupture remains constant following shut-in, while the average slip, and hence the magnitude of event, steadily decrease as the increased pore pressures diffuse into the surrounding fracture network. At the end of the 200-minute simulation, the results predict $18.5 \mathrm{~cm}$ slip and a $M_{w} 4.16$ event.

The magnitude versus time plot for the base case is compared with the simulation results from the parametric analysis in Figure 4 and Figure 5. For all simulations, the predicted magnitude of an induced event initially steeply increases with injection time. The rate of increase slowly declines to a peak magnitude, following which the predicted magnitude gradually decreases. The peak magnitude event is predicted at the end of the injection period for all simulations except the model with a shorter distance between the fault and the injector (i.e. 50 m compared to $100 \mathrm{~m}$; dotted orange curve in Figure 4), where the magnitude reaches a peak 50 minutes after shut-in. The results show that the greatest peak magnitude event, 4.56 , is predicted for the model with a fault length of $1.5 \mathrm{~km}$ (solid red curved in Figure 4) and the lowest peak magnitude event, 3.97, is predicted for the model with a Young's modulus of $10 \mathrm{GPa}$ for the shale matrix (dotted purple curve in Figure 4). The parameters with the greater impact on the peak magnitude are the Young's modulus (purple curves in Figure 4 and difference in magnitude of 0.48 between end-member models in Figure 5) and 
the length of the fault (0.47; red curves), followed by the rate of injection $(0.40$; grey curves), the duration of injection ( 0.30 ; light grey curves), and the distance from fault to injection source ( 0.24 ; orange curves). The parameters investigated with the lowest impact on the peak magnitude are the pore pressure $(0.016$; green curves in Figure 4), Poisson's ratio (0.021; pink curves), maximum principal stress (0.063; blue curves), and minimum principal stress (0.52; light blue curves). The pore pressure, minimum principal stress, and Poisson's ratio; however, are the parameters with the greatest impact on the change in magnitude with time during the post-injection period. A more detailed comparison of the models is provided below.
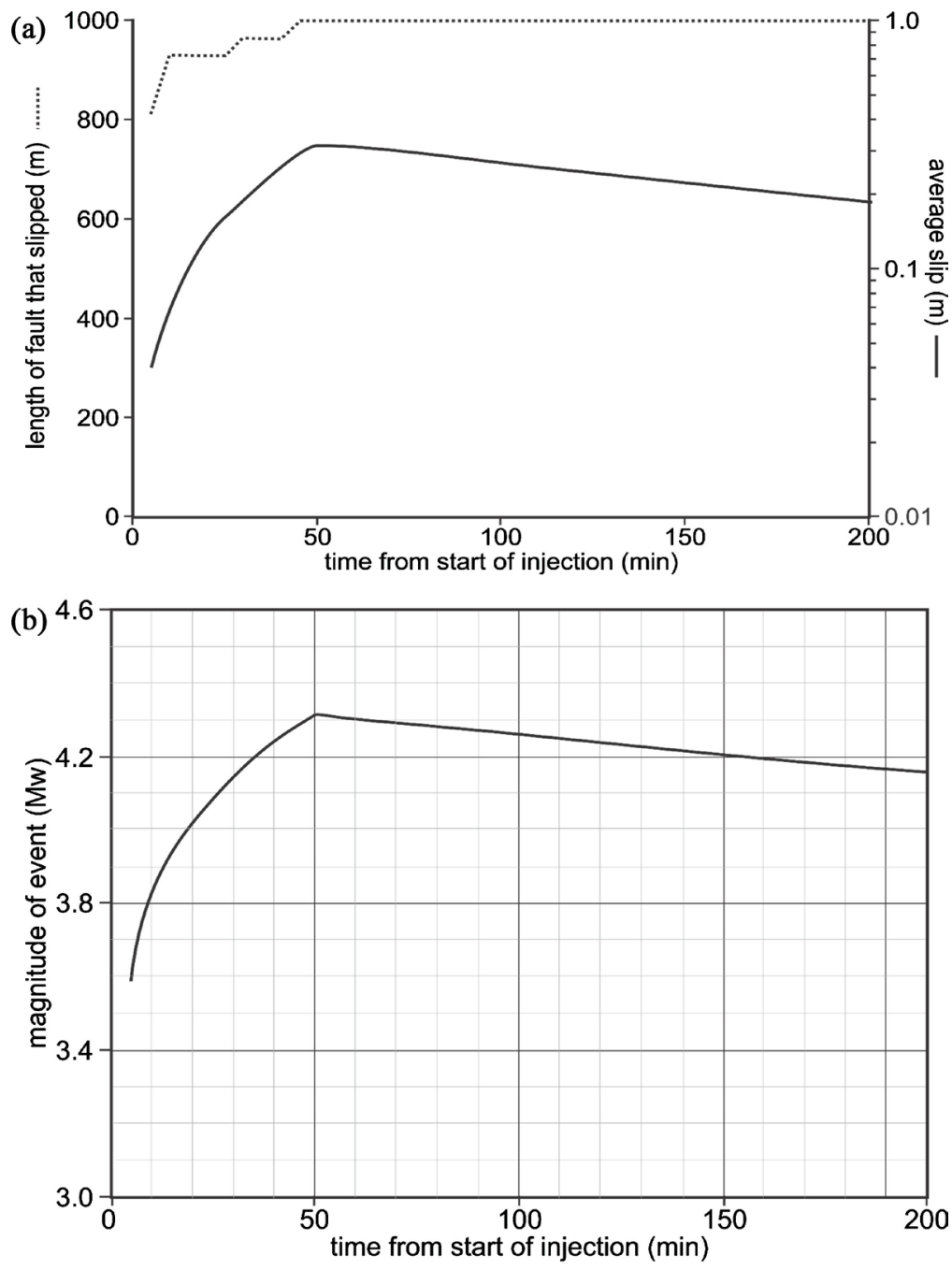

Figure 3. (a) The length of fault that slipped $>1 \mathrm{~cm}$ (i.e. fault rupture length; dotted curve) and the average shear slip along that length (i.e. average slip; solid curve) determined at 5-minute intervals during the 50-minute injection period and 10-minute intervals after shut-in by assuming all the slip along the fault was accommodated at that time. (b) The moment magnitude $\left(M_{w}\right)$ calculated from the modeled rupture length and slip with injection time in minutes for the base model with average parameter values. The largest magnitude event that could occur is a $M_{w} 4.31$ at the end of the 50-minute injection period. 


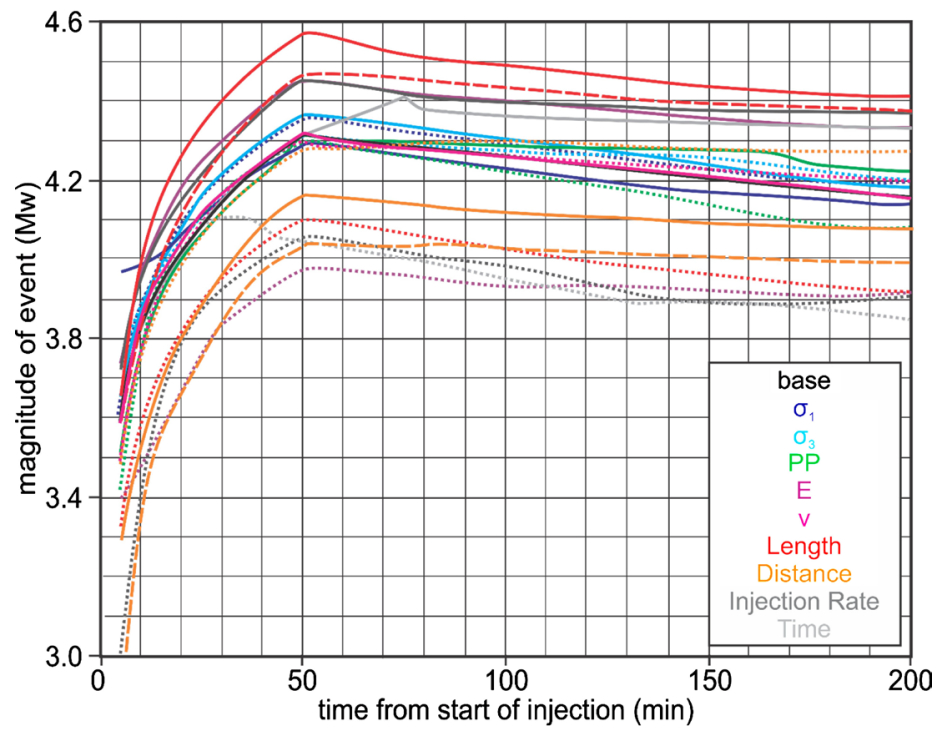

Figure 4. The calculated moment magnitude $\left(M_{w}\right)$ from the modeled rupture length and slip with injection time in minutes for the base model (solid black curve) compared to the results for the series of simulations from the parametric analysis. Maximum principal stress, $\sigma_{1}$ (dark blue curves); minimum principal stress, $\sigma_{3}$ (light blue curves); pore pressure, PP (green curves); Young's modulus, E (purple curves); Poisson's ratio, v (pink curves); fault length, Length (red curves); distance from fault to injector, Distance (orange curves); injection rate (dark grey curves); injection duration, Time (light grey curves).

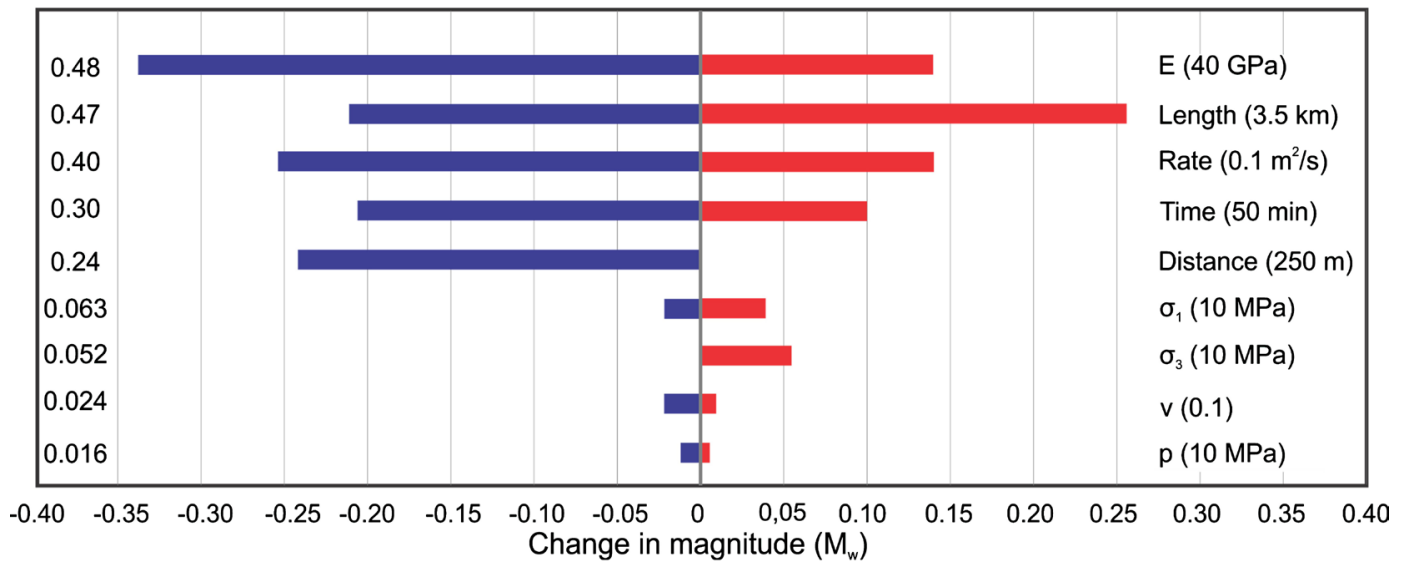

Figure 5. The increase (red) and decrease (blue) in the moment magnitude when the parameter values are changed from the values in the base case to the end-member values. The total change in magnitude between end-member values is indicated on the left hand side of plot and the parameters are listed in order of greatest change to the lowest with the change in parameter between end-member values shown in brackets. E: Young's modulus; Length: fault length; Rate: flow rate; Distance: distance from fault to injector; $\sigma_{1}$ : maximum principal stress; $\sigma_{3}$ : minimum principal stress, $v$. Poisson's ratio; $p$ : pore pressure.

\subsection{Rock Mechanics of the Shale}

Varying the rock mechanics properties of the shale reservoir has very little impact on the amount of slip or the rupture length of the fault (Figure 6 and Figure 7). The results show that weaker shales (the model with a Young's modulus of $10 \mathrm{GPa}$ and the model with a Poisson's ratio of 0.30 ) predict slightly larger slip 


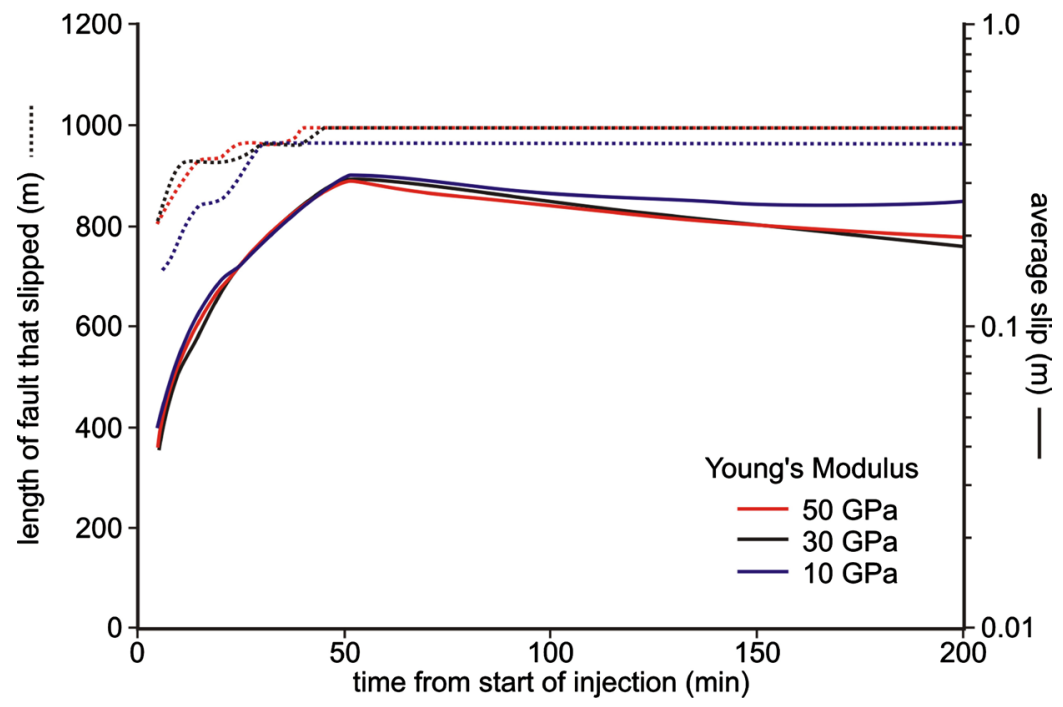

Figure 6. The length of fault that slipped $>1 \mathrm{~cm}$ (i.e. fault rupture length; dotted curve) and the average shear slip along that length (i.e. average slip; solid curve) versus injection time in minutes for the base model (black) compared to models with Young's moduli of $50 \mathrm{MPa}$ (red) and $10 \mathrm{MPa}$ (blue).

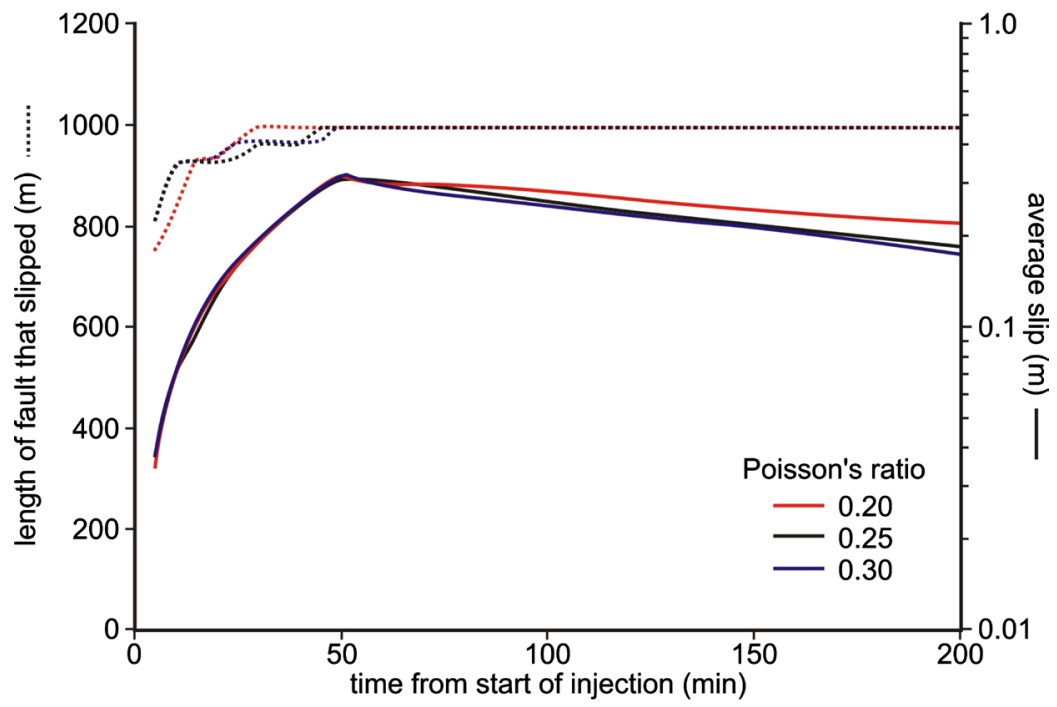

Figure 7. The length of fault that slipped $>1 \mathrm{~cm}$ (i.e. fault rupture length; dotted curve) and the average shear slip along that length (i.e. average slip; solid curve) versus injection time in minutes for the base model (black) compared to models with Poisson's ratios of $0.20 \mathrm{MPa}$ (red) and 0.30 MPa (blue).

over slightly shorter fault lengths than stronger shales. The Young's modulus; however, has a large impact on the shear modulus input into the calculation of the moment magnitude. The difference in the shear modulus results in the greatest difference in peak magnitude between end-member values for the parameters tested (Figure 4 and Figure 5). The stronger, more brittle model, with a Young's modulus of $50 \mathrm{MPa}$, predicts a $M_{w} 4.45$ peak induced event (solid red curve in Figure 8) compared to a $M_{W} 3.98$ peak event predicted by the model with a Young's modulus of $10 \mathrm{MPa}$ (dotted red curve in Figure 8). 


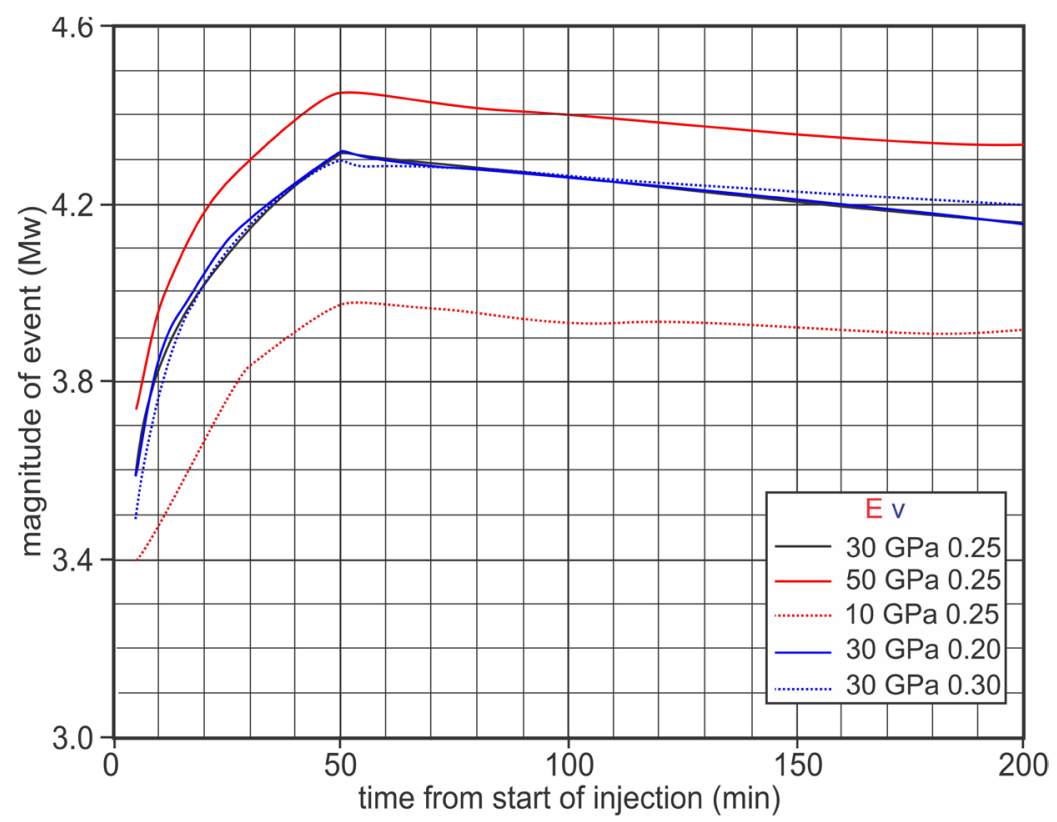

Figure 8. The calculated moment magnitude $\left(M_{w}\right)$ from the modeled rupture length and slip with injection time in minutes for the base model (black) compared to models with varying Young's modulus (E; red) and Poisson's ratio (v; blue) between end-member values.

\subsection{Length of Fault}

Three models were simulated with fault lengths of 500,1500, and $5000 \mathrm{~m}$ for comparison with the base model with a fault length of $1000 \mathrm{~m}$. The model with the $500 \mathrm{~m}$ long fault predicts the largest slip (solid blue curve in Figure 9), but the shortest fault rupture length (dotted blue curve on Figure 9), resulting in the lowest peak magnitude event of, $M_{w} 4.10$ (dotted red curve in Figure 10). The model with the $1500 \mathrm{~m}$ fault predicts smaller slip (solid red curve in Figure 9), but longer fault rupture length (dotted red curve in Figure 9) than the models with shorter faults (i.e. 500 and $1000 \mathrm{~m}$ ) resulting in a greater magnitude event of $M_{w} 4.56$ (solid red curve in Figure 10). However, while the model with the longest fault length of $5000 \mathrm{~m}$ predicts the longest fault rupture length (dotted green curve in Figure 9), the average slip along the fault is much smaller (solid green curve in Figure 9), resulting in a lower magnitude peak event, $M_{w} 4.46$ (dashed red curve in Figure 10), than for the model with a $1500 \mathrm{~m}$ fault. The results indicate that, for the tested model parameters, the magnitude of an induced event increases with fault length, up to a maximum length, after which the smaller slip along longer faults results in smaller magnitude events. Therefore, for the model in this study, the greatest hazard is posed by the moderate sized faults, $\sim 1-5 \mathrm{~km}$ in length.

\subsection{Distance to Injection Source}

Increasing the distance from the injection source to the center of the fault from $100 \mathrm{~m}$ in the base model to $200 \mathrm{~m}$ and $300 \mathrm{~m}$ predicts smaller slips (red and green versus black solid curves in Figure 11) along shorter fault lengths (red and 


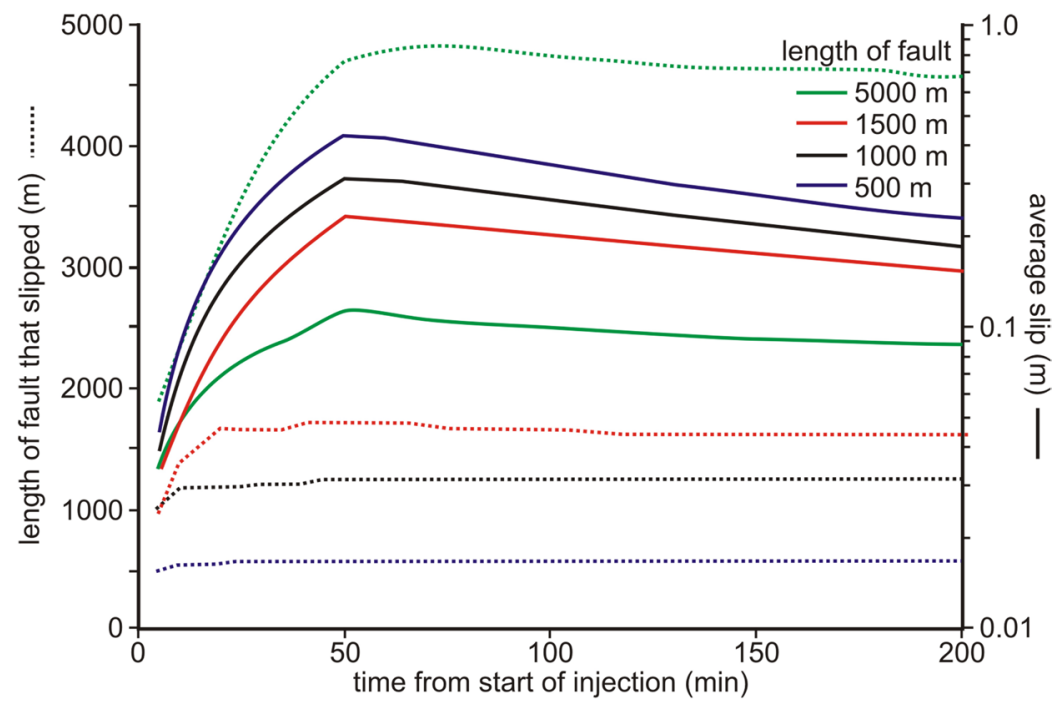

Figure 9. The length of fault that slipped $>1 \mathrm{~cm}$ (i.e. fault rupture length; dotted curve) and the average shear slip along that length (i.e. average slip; solid curve) versus injection time in minutes for the base model (black) compared to models with faults lengths of 500 $\mathrm{m}$ (blue), $1500 \mathrm{~m}$ (red), and $5000 \mathrm{~m}$ (green).

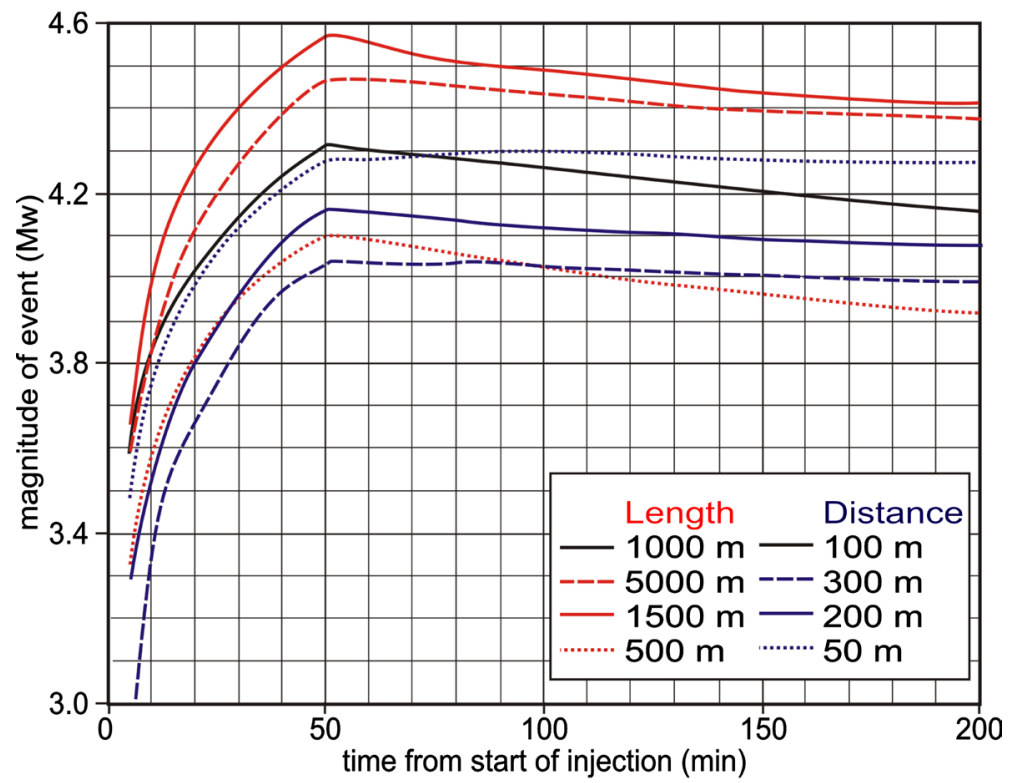

Figure 10. The calculated moment magnitude $\left(M_{w}\right)$ from the modeled rupture length and slip with injection time in minutes for the base model (black) compared to models with faults lengths of $500 \mathrm{~m}$ (dotted red), $1500 \mathrm{~m}$ (solid red), and $5000 \mathrm{~m}$ (dashed red) and models with distances from fault to injector of $50 \mathrm{~m}$ (dotted blue), $200 \mathrm{~m}$ (solid blue), and $300 \mathrm{~m}$ (dashed blue).

green versus black dotted curves in Figure 11), resulting in lower peak magnitude events of $M_{w} 4.16$ and 4.04 (solid and dashed blue curves in Figure 10). The interaction of the hydraulic and natural fractures with the fault in the model with an injection source only $50 \mathrm{~m}$ from the fault predicts smaller slip (blue versus black solid curves in Figure 11) resulting in a lower magnitude event of $M_{w}$ 4.27 than for the base model (dotted blue versus solid black curves in Figure 10). 


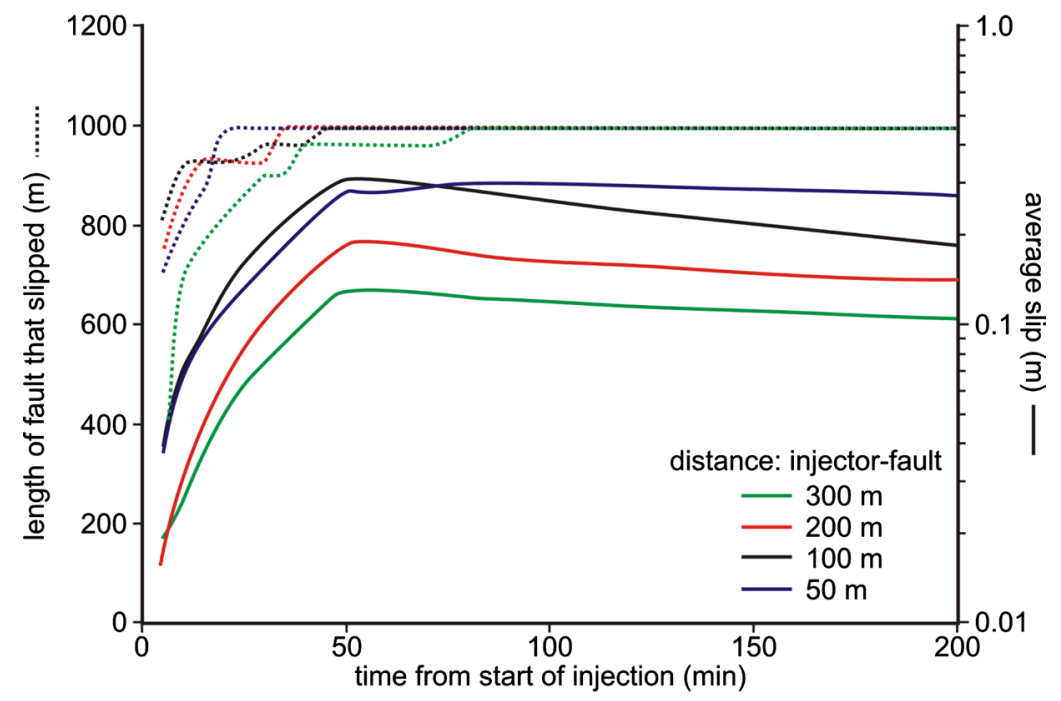

Figure 11. The length of fault that slipped $>1 \mathrm{~cm}$ (i.e. fault rupture length; dotted curve) and the average shear slip along that length (i.e. average slip; solid curve) versus injection time in minutes for the base model (black) compared to models with distances from fault to injector of $50 \mathrm{~m}$ (blue), $200 \mathrm{~m}$ (red), and $300 \mathrm{~m}$ (green).

The model with a $50 \mathrm{~m}$ distance between injector and fault is the only model in the study where the average slip, and hence magnitude, continues to increase post-injection. The greatest magnitude event predicted for the model with a 50 m distance is a $M_{w} 4.30$ event, 50 minutes after shut-in.

\subsection{Duration and Rate of Fluid Injection}

The fluid injection rate of $0.1 \mathrm{~m}^{2} / \mathrm{s}$ over 50 minutes for the base model results in $300 \mathrm{~m}^{2}$ of injected fluid during the simulation. Increasing the amount of injected fluid to $450 \mathrm{~m}^{2}$, by increasing the injection duration to $75 \mathrm{~min}$, delays and increases the peak magnitude of an event from 4.31 to 4.41 (solid blue curve in Figure 12). Decreasing the amount of fluid injected to $150 \mathrm{~m}^{2}$, by decreasing the injection duration to $25 \mathrm{~min}$, results in an earlier, lower magnitude peak event of $M_{w} 4.10$ (dotted blue curve in Figure 12). Varying the amount of fluid injected from $450 \mathrm{~m}^{2}$ to $150 \mathrm{~m}^{2}$, by changing the injection rate from $0.15 \mathrm{~m}^{2} / \mathrm{s}$ to 0.05 $\mathrm{m}^{2} / \mathrm{s}$ results in peak magnitudes of 4.45 (solid red curve in Figure 12) and 4.06 (dotted red curve in Figure 12). The injection rate; therefore, has a greater impact on the magnitude than the injection duration. The results also show that a lower magnitude event is predicted later in time if the same amount of fluid is injected with a slower rate over a longer duration. The entire length of the $1 \mathrm{~km}$ long fault is not predicted to significantly rupture until shut-in for the two models with $150 \mathrm{~m}^{2}$ of injected fluid (dotted, blue and light blue curves in Figure 13).

\subsection{Effective Principal Stresses}

As a result of the initial critical stress state of the fault in the models, varying the effective principal stresses by $5 \mathrm{MPa}$ has a lower impact on the predicted magnitude of induced event, in comparison to the other model parameters tested. The 


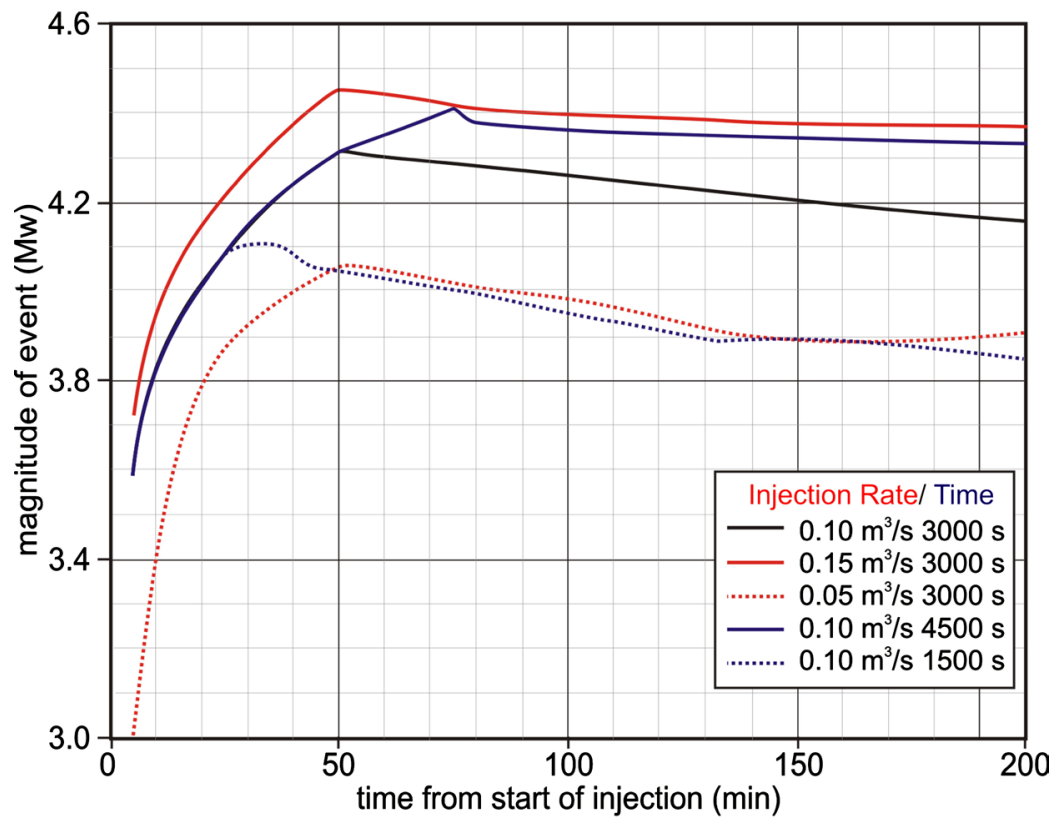

Figure 12. The calculated moment magnitude $\left(M_{w}\right)$ from the modeled rupture length and slip with injection time in minutes for the base model (black) compared to models with varying injection rate (red) and injection duration (time; blue) between end-member values.

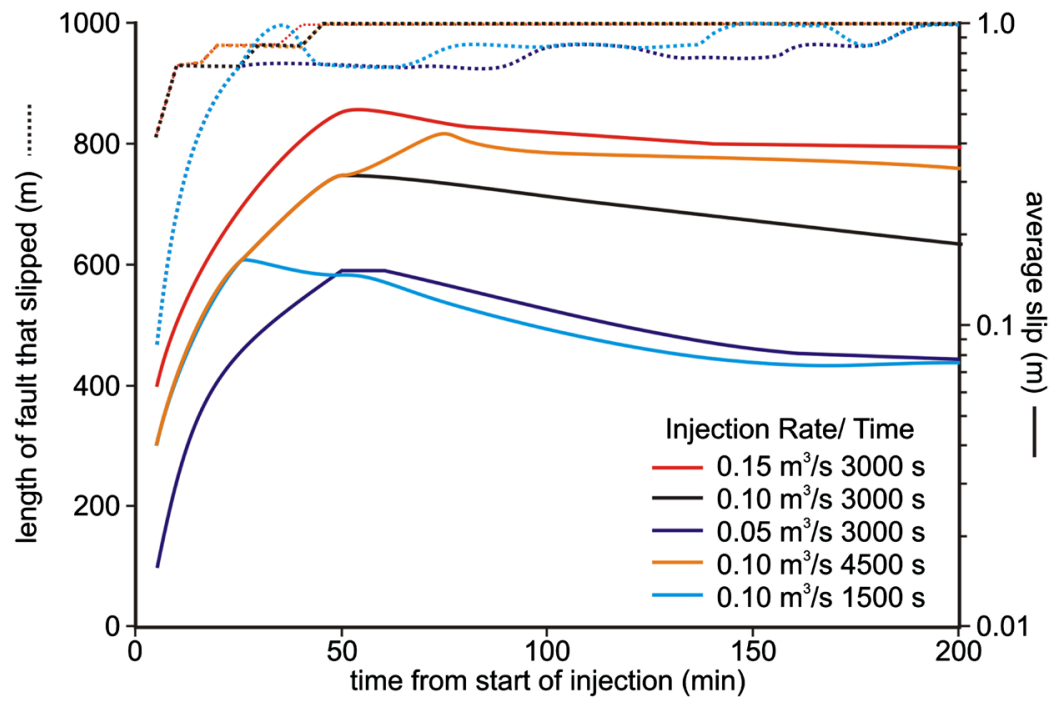

Figure 13. The length of fault that slipped $>1 \mathrm{~cm}$ (i.e. fault rupture length; dotted curve) and the average shear slip along that length (i.e. average slip; solid curve) versus injection time in minutes for the base model (black) compared to models with varying injection rate (red and orange) and duration (blue and light blue) between end-member values.

results show that the peak magnitude is least sensitive to the pore pressure of all parameters tested (Figure 4 and Figure 5). Increasing and decreasing the initial pore pressure by $5 \mathrm{MPa}$ changes the peak magnitude from 4.31 to 4.30 (solid and dotted green curves in Figure 14). Higher initial pore pressures (i.e. $15 \mathrm{MPa}$ ) predict larger slip (solid red curve in Figure 15); however, shorter fault rupture lengths (dotted red curve in Figure 15), which trade-off resulting in a low impact 


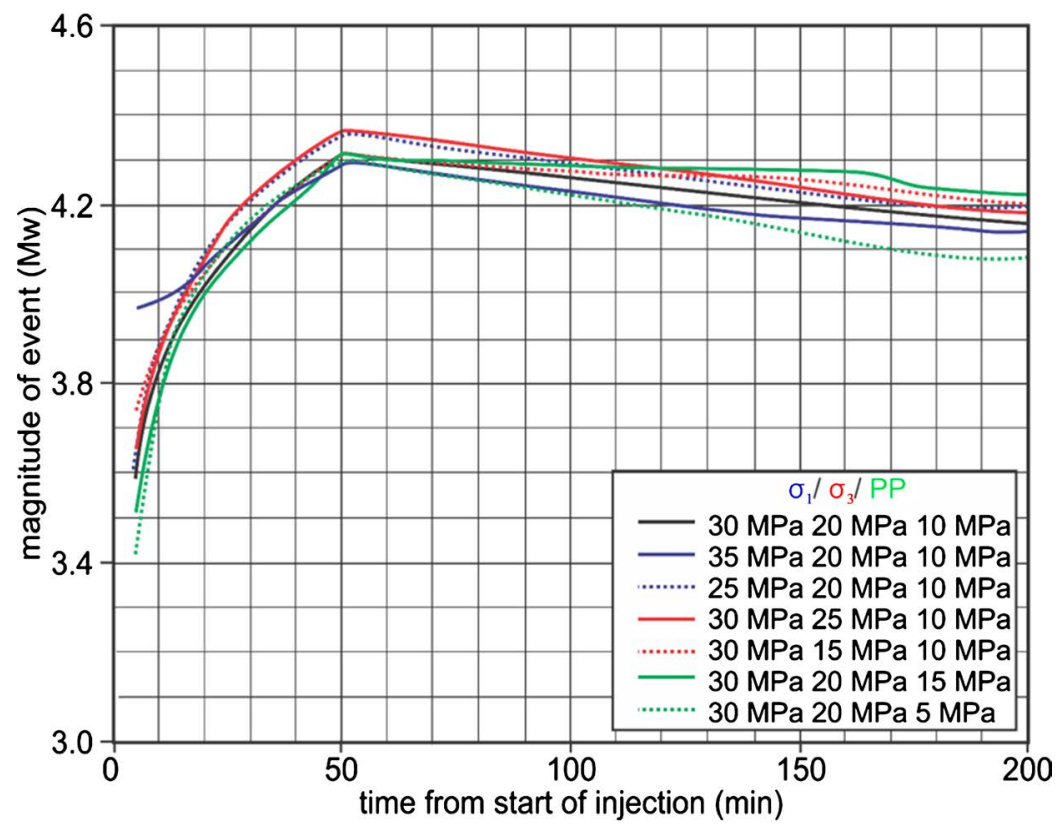

Figure 14. The calculated moment magnitude $\left(M_{w}\right)$ from the modeled rupture length and slip with injection time in minutes for the base model (black) compared to models with varying maximum principal stress $\left(\sigma_{1}\right.$; blue $)$, minimum principal stress $\left(\sigma_{3} ;\right.$ red), and pore pressure (PP; green) between end-member values.

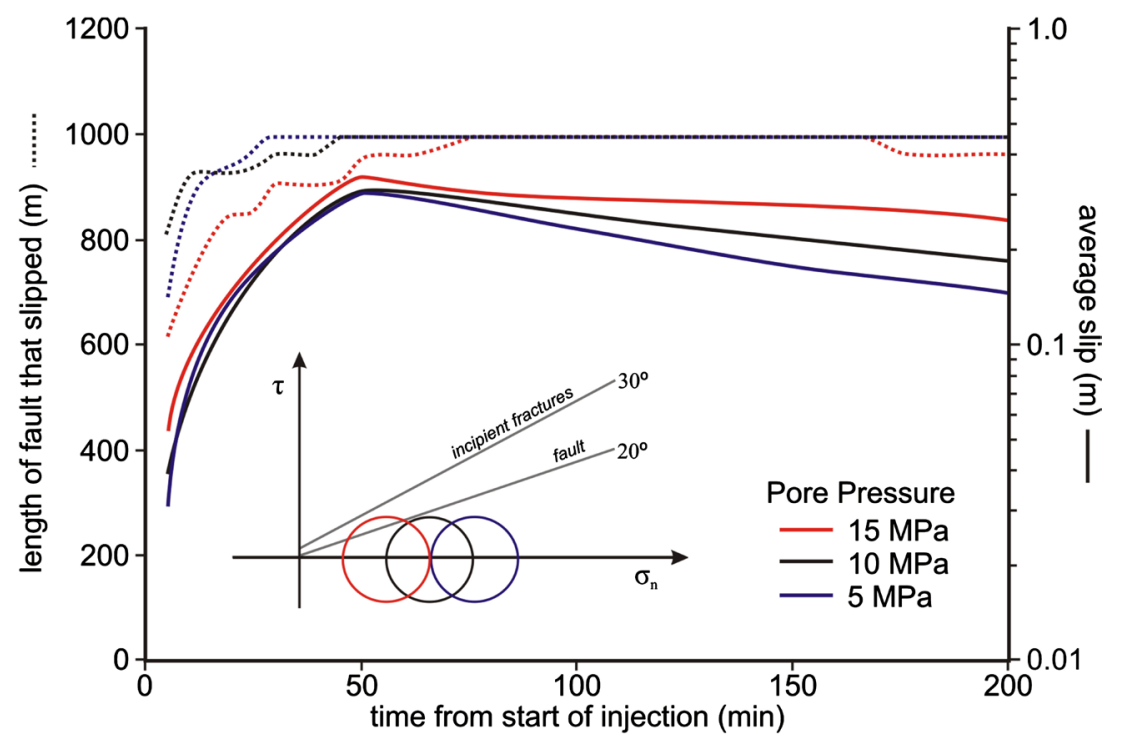

Figure 15. The length of fault that slipped $>1 \mathrm{~cm}$ (i.e. fault rupture length; dotted curve) and the average shear slip along that length (i.e. average slip; solid curve) versus injection time in minutes for the base model (black) compared to models with pore pressures of 15 $\mathrm{MPa}$ (red) and $5 \mathrm{MPa}$ (blue).

on the magnitude of induced event.

Increasing the differential stress, by increasing the maximum principal stress to $35 \mathrm{MPa}$ from $30 \mathrm{MPa}$ in the base model, predicts a lower peak magnitude event of $M_{W} 4.29$ (solid blue curve in Figure 14), while decreasing the differential stress, by decreasing the maximum principal stress to $25 \mathrm{MPa}$, predicts a greater 
peak magnitude event of $M_{w} 4.35$ (dotted blue curve in Figure 14). Similarly, increasing the differential stress by, decreasing the minimum principal stress to 15 $\mathrm{MPa}$ from $20 \mathrm{MPa}$ in the base model, predicts a slightly lower peak magnitude event (dotted red curve in Figure 14), while decreasing the differentail stress, by increasing the minimum principal stress to $25 \mathrm{MPa}$, predicts a greater peak magnitude event of $M_{W} 4.36$ (solid red curve in F Figure 14). Such results may seem contrary to intuition and previous results from numerical studies, which found that higher differential stresses likely to result in large events. However, in this study, higher differential stresses result in failure of the incipient natural fractures in the models. A failure state for the incipient fractures is predicted before injection for the model with a minimum principal stress of $15 \mathrm{MPa}$ (blue Mohr circle on inset plot in Figure 16) and with a small pore pressure increase for the model with a maximum principal stress of $35 \mathrm{MPa}$ (blue Mohr circle on inset plot in Figure 17). The failure of the incipient fractures dissipates the stress and pore pressures resulting in smaller increases in stress along the fault plane and therefore lower magnitude events. In contrast, the incipient fractures remain intact for the models with lower differential stresses, resulting in a larger pore pressure increase along the fault and thus greater slip and magnitude events.

The models with critically stressed fractures resulting from the effective principal stresses (the model with a pore pressure of $15 \mathrm{MPa}$, the model with a minimum principal stress of $15 \mathrm{MPa}$, and the model with a maximum principal stress of $35 \mathrm{MPa}$ ) predict greater pore pressure diffusion following shut-in, resulting in a more gradual decrease in the slip (solid red curve in Figure 15, solid blue curve in Figure 16, and solid blue curve in Figure 17) and hence the predicted magnitude of an induced event with time (solid green curve, dotted red curve, and solid blue curve in Figure 14).

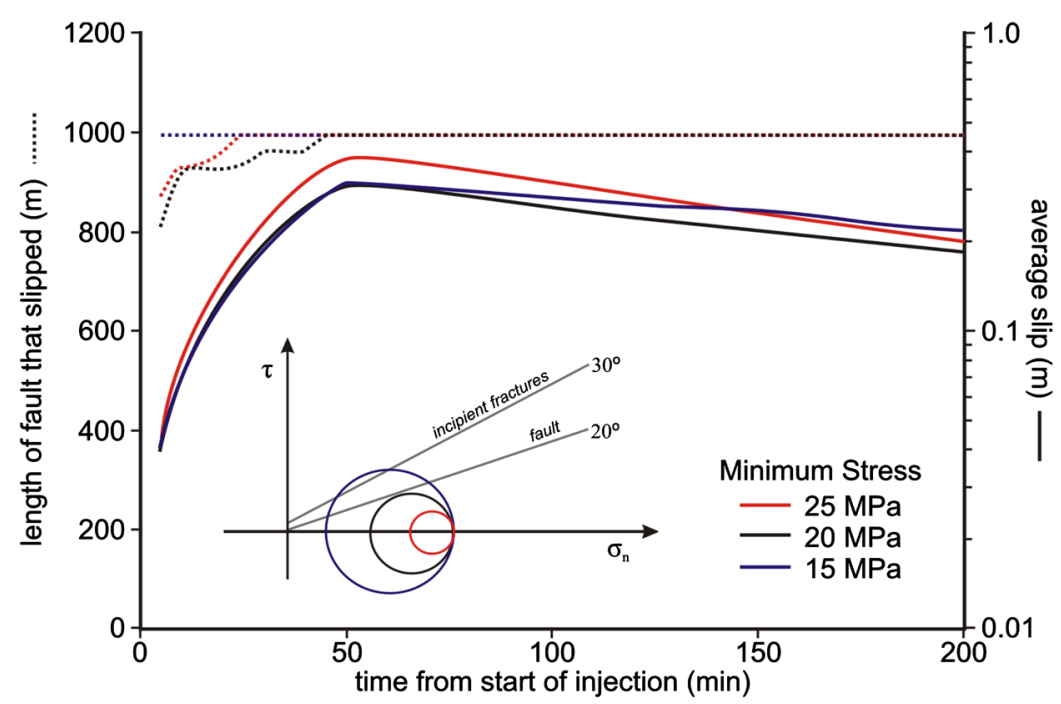

Figure 16. The length of fault that slipped $>1 \mathrm{~cm}$ (i.e. fault rupture length; dotted curve) and the average shear slip along that length (i.e. average slip; solid curve) versus injection time in minutes for the base model (black) compared to models with minimum principal stresses of $25 \mathrm{MPa}$ (red) and $15 \mathrm{MPa}$ (blue). 


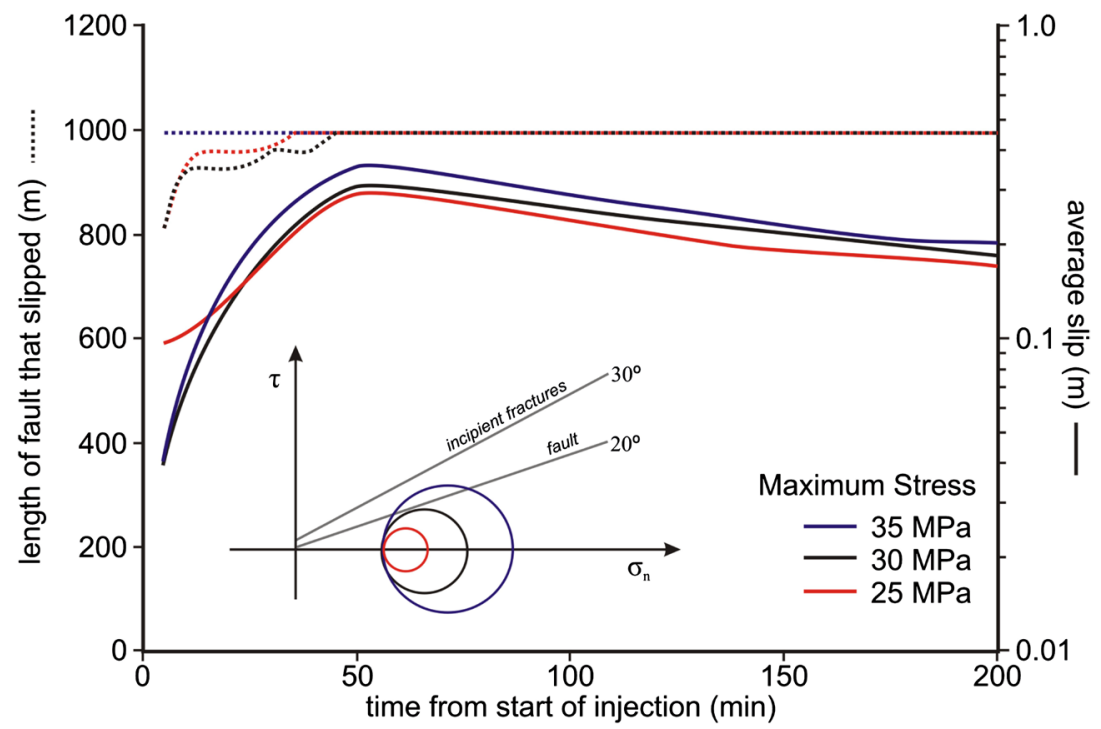

Figure 17. The length of fault that slipped $>1 \mathrm{~cm}$ (i.e. fault rupture length; dotted curve) and the average shear slip along that length (i.e. average slip; solid curve) versus injection time in minutes for the base model (black) compared to models with maximum principal stresses of $25 \mathrm{MPa}$ (red) and $35 \mathrm{MPa}$ (blue).

\section{Conclusions}

A series of 2D hydro-geomechanical models, representing potential worst-case scenarios where fluid is injected by a hydraulic fracturing stage nearby a permeable, brittle, critically-stressed fault, were simulated and compared to provide insights into the sensitivity of the magnitude of seismicity induced by hydraulic fracturing. The sensitivity of the magnitude to fluid injection rate and duration, fault length and its distance to the injector, effective stress state, and shale geomechanics were quantitatively compared through a parametric analysis.

The base model for the parametric analysis, with fluid injected at a rate of 0.1 $\mathrm{m}^{2} / \mathrm{s}$ for $50 \mathrm{~min}, 100 \mathrm{~m}$ from the centre of a $1 \mathrm{~km}$ long fault predicts a maximum event with $M_{w}$ 4.31. A quantitative comparison of the simulation results show that the magnitude of earthquake induced by a hydraulic fracturing stage ranges from 3.97 to 4.56 . The model with a longer fault of $1.5 \mathrm{~km}$, predicts the greatest magnitude and the model with a smaller Young's modulus (10 versus $30 \mathrm{MPa}$ ) predicts the lowest magnitude. The differences in magnitude predicted for end-member parameter values indicate that the Young's modulus (difference of 0.48 ) and the length of the fault (difference of 0.47 ) have the greatest impact on the magnitude of the parameters tested. The tested parameter with the next greatest impact on the magnitude is the rate of injection (difference of 0.4 ) followed by the duration of injection (difference of 0.30 ) and then the distance from the fault to the injection source (difference 0.24). In comparison, the pore pressure (difference of 0.016), Poisson's ratio (difference of 0.021), maximum principal stress (difference of 0.063), and minimum principal stress (difference of 0.052) have little impact on the magnitude of induced event.

Understanding the maximum possible magnitude of seismicity induced by 
hydraulic fracturing and the sensitivity of the magnitude to geologic structure, rock mechanical properties, and completions parameters will aid in developing protocols for reducing the probability of inducing felt events and mitigation procedures to prevent larger magnitude induced events, as well as help to understand the variations in the magnitude of induced seismicity between regions.

\section{Conflicts of Interest}

The authors declare no conflicts of interest regarding the publication of this paper.

\section{References}

[1] de Pater, C.J. and Baisch, S. (2011) Geomechanical Study of Bowland Shale Seismicity.

https://www.rijksoverheid.nl/binaries/rijksoverheid/documenten/rapporten/2011/1 1/04/rapport-geomechanical-study-of-bowland-shale-seismicity/rapport-geomecha nical-study-of-bowland-shale-seismicity.pdf

[2] Bustin, R.M. and Bustin, A.M.M. (2012) Induced Seismicity in the Bowland Basin, during Completion of the Preese Hall Shale Gas/Oil Well. SPE/SGE Workshop on Injection Induced Seismicity, Broomfield, September 2012.

[3] Clarke, H., Eisner, L., Styles, P. and Turner, P. (2014) Felt Seismicity Associated with Shale Gas Hydraulic Fracturing: The First Documented Example in Europe. Geophysical Research Letters, 41, 8308-8314. https://doi.org/10.1002/2014GL062047

[4] Hallo, M.I., Oprsal, L., Eisner, L. and Ali, M.Y. (2014) Prediction of Magnitude of the Largest Potentially Induced Seismic Event. Journal of Seismology, 18, 421-431. https://doi.org/10.1007/s10950-014-9417-4

[5] McGarr, A. (2014) Maximum Magnitude Earthquakes Induced by Fluid Injection. Journal of Geophysical Research: Solid Earth, 119, 1008-1019. https://doi.org/10.1002/2013JB010597

[6] McGarr, A. and Barbour, A.J. (2018) Injection-Induced Moment Release Can Also Be Aseismic. Geophysical Research Letters, 45, 5344-5351. https://doi.org/10.1029/2018GL078422

[7] Shapiro, S.A., Kruger, O.S., Dinske, C. and Langenbruch, C. (2011) Magnitudes of Induced Earthquakes and Geometric Scales of Fluid-Stimulated Rock Volumes. Geophysics, 76, WC55-WC63. https://doi.org/10.1190/geo2010-0349.1

[8] Galis, M., Ampuero, J.P., Mai, P.M. and Cappa, F. (2017) Induced Seismicity Provides Insight into Why Earthquake Ruptures Stop. Science Advances, 3, eaap7528. https://doi.org/10.1126/sciadv.aap7528

[9] Maurer, J. and Segall, P. (2018) Magnitudes of Induced Earthquakes in Low-Stress Environments. Bulletin of the Seismological Society of America, 108, 1087-1106. https://doi.org/10.1785/0120170295

[10] Itasca Consulting Group Inc. (2011) UDEC (Universal Distinct Element Code), Version 5.0. ICG, Minneapolis.

[11] Cundall, P.A. (1971) A Compute Model for Simulating Progressive Large Scale Movements in Blocky Rock Systems. Proceedings of the Symposium of the International Society for Rock Mechanics, Nancy, Vol. 1, II-8.

[12] Hanks, T.C. and Kanamori, H. (1979) Moment Magnitude Scale. Journal of Geo- 
physical Research, 84, 2348-2350. https://doi.org/10.1029/JB084iB05p02348

[13] Aki, K. (1966) Generation and Propagation of G Waves from the Niigata Earthquake of June 14, 1964. Part 2. Estimation of Earthquake Moment, Released Energy and Stress-Strain Drop from G Wave Spectrum. Bulletin of the Earthquake Research Institute, 44, 73-88.

[14] Rutqvist, J., Cappa, F., Rinaldi, A.P. and Godano, M. (2014) Dynamic Modeling of Injection-Induced Fault Reactivation and Ground Motion and Impact on Surface Structures and Human Perception. Energy Procedia, 63, 3379-3389. https://doi.org/10.1016/j.egypro.2014.11.367

[15] Rutqvist, J., Rinaldi, A.P., Cappa, F. and Moridis, G.J. (2015) Modeling of Fault Activation and Seismicity by Injection Directly into a Fault Zone Associated with Hydraulic Fracturing of Shale-Gas Reservoirs. Journal of Petroleum Science and Engineering, 127, 377-386. https://doi.org/10.1016/j.petrol.2015.01.019 